\title{
Boundary terms in the decomposition of nucleon spin
}

\section{Peter LOWDON*}

Universität Zürich (CH)

E-mail: lowdon@physik.uzh.ch

The decomposition of nucleon spin into internal and orbital angular momentum contributions from partons is revisited in the context of quantum field theory. It is shown that commonly used decompositions rely on classical-type arguments for their justification, and that these arguments may no longer hold in the full quantum theory, in particular with respect to the treatment of boundary terms. The role of these terms in the construction of quark-gluon decompositions of the QCD angular momentum operator is investigated, casting doubt on the applicability of certain identifications of quark and gluon contributions to the nucleon spin.

XXIII International Workshop on Deep-Inelastic Scattering 27 April - May 12015

Dallas, Texas

* Speaker. 


\section{Introduction}

Understanding the spin structure of nucleons remains an important unresolved problem in QCD. A major focus of investigations in recent years has centred around settling the so-called spin crisis, which refers to results obtained by the European Muon Collaboration (EMC) [1] that appeared to suggest that quarks accounted for a relatively small amount of the spin of the proton. This seemingly surprising result has since led to many proposed solutions, a significant proportion of which involve splitting the QCD angular momentum operator up in different ways, and arguing a particular physical interpretation of the resulting pieces. It turns out that spatial boundary operators play a particularly important role in these decompositions $[2,3,4,5,6]$, and yet the treatment of these terms is often justified using classical-type arguments, which may no longer hold in the physical quantised theory. In these proceedings, we summarise an approach [7] which aims to address these issues from a more rigorous quantum field theory (QFT) perspective.

\section{The QCD angular momentum decomposition}

To discuss the issues related to the decompositions of the QCD angular momentum operator $J_{Q C D}^{i}$ proposed in the literature $[2,3,4,5,6]$, one must first determine the structure of the QCD Lorentz current $M_{Q C D}^{\mu \nu \lambda}$. In one of the first discussions of possible $J_{Q C D}^{i}$ decompositions in the literature [2], it was shown that it is possible to write this current in the following way:

$$
\begin{aligned}
M_{Q C D}^{\mu \nu \lambda}= & \frac{i}{2} \bar{\psi} \gamma^{\mu}\left(x^{v} \partial^{\lambda}-x^{\lambda} \partial^{v}\right) \psi+\text { h.c. }+\frac{1}{2} \varepsilon^{\mu \nu \lambda \rho} \bar{\psi} \gamma_{\rho} \gamma^{5} \psi \\
& -F^{\mu \rho a}\left(x^{v} \partial^{\lambda}-x^{\lambda} \partial^{v}\right) A_{\rho}^{a}+F^{\mu \lambda a} A^{v a}+F^{v \mu a} A^{\lambda a}+\frac{1}{4} F_{\alpha \beta}^{a} F^{\alpha \beta a}\left(x^{v} g^{\mu \lambda}-x^{\lambda} g^{\mu v}\right) \\
& -\frac{i}{16} \partial_{\beta}\left[x^{v} \bar{\psi}\left\{\gamma^{\lambda},\left[\gamma^{\mu}, \gamma^{\beta}\right]\right\} \psi-(v \leftrightarrow \lambda)\right]+\partial_{\beta}\left(x^{v} F^{\mu \beta a} A^{\lambda a}-x^{\lambda} F^{\mu \beta a} A^{v a}\right)
\end{aligned}
$$

By applying the canonical definition for the angular momentum charge $J_{Q C D}^{i}:=\frac{1}{2} \varepsilon^{i j k} \int d^{3} x M_{Q C D}^{0 j k}$, $J_{Q C D}^{i}$ can then be written:

$$
\begin{aligned}
& J_{Q C D}^{i}=\underbrace{\varepsilon^{i j k} \int d^{3} x\left[\frac{i}{2} \bar{\psi} \gamma^{0}\left(x^{j} \partial^{k}\right) \psi+\text { h.c. }\right]}_{:=L_{q}^{i}}+\underbrace{\varepsilon^{i j k} \int d^{3} x\left(\frac{1}{4} \varepsilon^{0 j k l} \bar{\psi} \gamma_{l} \gamma^{5} \psi\right)}_{:=S_{q}^{i}} \\
& \underbrace{-\varepsilon^{i j k} \int d^{3} x\left[F^{0 l a}\left(x^{j} \partial^{k}\right) A_{l}^{a}\right]}_{:=L_{g}^{i}}+\underbrace{\varepsilon^{i j k} \int d^{3} x\left(F^{0 k a} A^{j a}\right)}_{:=S_{g}^{i}} \\
& \underbrace{-\frac{i}{16} \varepsilon^{i j k} \int d^{3} x \partial_{l}\left(x^{j} \bar{\psi}\left\{\gamma^{k},\left[\gamma^{0}, \gamma^{l}\right]\right\} \psi\right)}_{:=S_{1}^{i}}+\underbrace{\varepsilon^{i j k} \int d^{3} x \partial_{l}\left(x^{j} F^{0 l a} A^{k a}\right)}_{:=S_{2}^{i}}
\end{aligned}
$$

If one chooses to drop the spatial boundary operators $\mathcal{S}_{1}^{i}$ and $\mathcal{S}_{2}^{i}$, the resulting expression is referred to as the Jaffe-Manohar decomposition [6]. This process of decomposing the angular momentum operator into various pieces, and dropping spatial boundary terms, is in fact common to many of the decompositions proposed in the literature $[2,4,5,6]$. The advantage of this procedure is that 
one ends up with expressions where the individual terms involve either only quark or only gluon fields, which makes it suggestive to interpret each of these terms as corresponding to physically distinct angular momentum sources. However, the requirement that these operator decompositions hold depends solely on whether it is possible to ignore spatial boundary operators of the form:

$$
\int d^{3} x \partial_{i} B^{i}(x)
$$

The argument which is often used to justify dropping these terms is that one can invoke Stokes' Theorem, and by assuming that $B^{i}(x)$ vanishes at spatial infinity this then implies the vanishing of the operator. However, as will be discussed in section 3, these arguments in general no longer hold for a quantised field theory, and so the question of whether spatial boundary operators vanish is more subtle. Nevertheless, if one accepts the validity of these angular momentum decompositions, then one can form a matrix element sum rule by inserting a certain component of these operators between some physical polarised state. In the case of the proton spin sum rules, which are often used to explain the spin crisis observed by the EMC, the $z$-components of the operators in the $J_{Q C D}^{z}$ decomposition are inserted between $z$-polarised proton states. For the Jaffe-Manohar decomposition in particular, the matrix elements formed in this way from the operators $S_{q}^{z}, L_{q}^{z}$ and $S_{g}^{z}, L_{g}^{z}$ are interpreted as the contributions to the $z$-component of the internal spin and orbital angular momentum of the proton from the quarks $S_{q}, L_{q}$ and gluons $S_{g}, L_{g}$ respectively [2, 3]. Since the proton has a total angular momentum of one-half, the Jaffe-Manohar sum rule takes the following form:

$$
\frac{1}{2}=S_{q}+L_{q}+S_{g}+L_{g}
$$

where a sum over quark flavour is also implicitly contained in the terms $S_{q}$ and $L_{q}$. It is then often stated that the apparent smallness of $S_{q}$ measured by the EMC can be explained by the nonvanishing of the remaining terms. In other words, the rest of the spin of the proton comes from the internal spin of the gluons as well as the orbital angular momentum of the quarks and gluons.

\section{Spatial boundary operators}

As mentioned in section 2, the issue of when spatial boundary operators vanish in a QFT is more subtle than for the corresponding classical theory. The reason behind this is that quantised fields are not operator-valued functions as one might assume, but are in fact operator-valued distributions. Distributions, unlike functions, are in general not point-wise defined [8], which means that one cannot always guarantee that the requirement for a quantised field $B^{i}(x)$ to satisfy a boundary condition, such as to vanish at spatial infinity, always makes sense. Distributions by definition must also be smeared with some suitable test function $f$, which means that a quantised field $\varphi(x)$ is not by itself an operator, only the smeared expression: $\varphi(f):=\int d^{4} x \varphi(x) f(x)$ is. This approach of treating quantised fields as distributions is actually one of a series of axioms which are employed in rigorous formulations of QFT. Although different schemes have been proposed [8, 9, 10, 11], these approaches generally consist of a common core set of axioms, from which one can derive more general results. In the case of spatial boundary operators, it turns out that one can use this approach to determine both a necessary and sufficient condition on when these operators vanish in the Hilbert space of physical states $\mathscr{H}$ [7]: 
Theorem 1. $\quad \int d^{3} x \partial_{i} B^{i}$ vanishes in $\mathscr{H} \Longleftrightarrow \int d^{3} x \partial_{i} B^{i}|0\rangle=0$

The surprising conclusion of this theorem is that the vanishing of a spatial boundary operator only requires that the corresponding operator annihilates the vacuum state $|0\rangle$, and does not depend on how it acts on the full space of states. However, in order to practically determine whether this operator annihilates the vacuum or not, it is easier instead to consider equivalent matrix element conditions. These conditions have the following form [7]:

$$
\begin{aligned}
\text { If }\left\langle\Psi\left|\int d^{3} x \partial_{i} B^{i}\right| 0\right\rangle=0, \forall|\Psi\rangle \in \mathscr{H} & \Longrightarrow \int d^{3} x \partial_{i} B^{i}|0\rangle=0 \\
\text { If } \exists|\Psi\rangle \in \mathscr{H} \text { s.t. }\left\langle\Psi\left|\int d^{3} x \partial_{i} B^{i}\right| 0\right\rangle \neq 0 & \Longrightarrow \int d^{3} x \partial_{i} B^{i}|0\rangle \neq 0
\end{aligned}
$$

These relations imply that if one can find any state $|\Psi\rangle \in \mathscr{H}$ such that: $\left\langle\Psi\left|\int d^{3} x \partial_{i} B^{i}\right| 0\right\rangle \neq 0$, then this definitively proves that: $\int d^{3} x \partial_{i} B^{i}|0\rangle \neq 0$, and hence by Theorem 1: $\int d^{3} x \partial_{i} B^{i} \neq 0$. Otherwise, it must be the case that: $\int d^{3} x \partial_{i} B^{i}|0\rangle=0$, and thus: $\int d^{3} x \partial_{i} B^{i}=0$. Using the transformation property of fields under translations, it then follows [7] that the vanishing or non-vanishing of spatial boundary operators can be directly attributed to the vanishing or non-vanishing of the matrix elements $\left\langle\Psi\left|B^{i}(0)\right| 0\right\rangle$.

\section{Boundary terms in the proton spin sum rule}

In section 2 it was outlined that in order for angular momentum operator decompositions to hold in general, one is required to drop certain spatial boundary operators. In the case of the Jaffe-Manohar decomposition, these operators $\mathcal{S}_{1}^{i}$ and $\mathcal{S}_{2}^{i}$ have the following form:

$$
\mathcal{S}_{1}^{i}=-\frac{i}{16} \varepsilon^{i j k} \int d^{3} x \partial_{l}\left(x^{j} \bar{\psi}\left\{\gamma^{k},\left[\gamma^{0}, \gamma^{l}\right]\right\} \psi\right) \quad \mathcal{S}_{2}^{i}=\varepsilon^{i j k} \int d^{3} x \partial_{l}\left(x^{j} F^{0 l a} A^{k a}\right)
$$

Using the results of section 3, one can now test whether or not these operators vanish, and hence whether this operator decomposition holds. The simplest case is to choose the state $|\Psi\rangle$ in conditions 3.1 and 3.2 to be the vacuum state $|0\rangle$. The corresponding matrix elements in this case are then proportional to the vacuum expectation values $\left\langle 0\left|F^{0 j a} A^{k a}\right| 0\right\rangle$ and $\left\langle 0\left|\bar{\psi} \gamma^{l} \gamma^{5} \psi\right| 0\right\rangle$. Although the first of these condensates has to our knowledge not been determined before in the literature, the second condensate has, and there is evidence to suggest that it may be non-vanishing [12]. From the discussion in section 3 this then suggests that $S_{1}^{i}$ is non-vanishing, and therefore casts doubt on the validity of the Jaffe-Manohar angular momentum operator decomposition: $J_{Q C D}^{i}=S_{q}^{i}+L_{q}^{i}+S_{g}^{i}+L_{g}^{i}$.

Often in the derivation of proton spin sum rules it is firstly assumed that the decompositions of $J_{Q C D}^{z}$ hold exactly on an operator level, and then the decomposed expression for $J_{Q C D}^{z}$ is inserted between polarised proton states. But it seems, as demonstrated in the case of the Jaffe-Manohar decomposition, that this reasoning may not necessarily be correct. Nevertheless, it may be the case that the corresponding spatial boundary operators are in general non-vanishing, but do vanish when inserted between the proton states, and thus the proton spin sum rule itself continues to hold. However, it appears for the Jaffe-Manohar decomposition as though this is not the case [7], and 
therefore that the Jaffe-Manohar sum rule in equation 2.4 may not actually hold. It is interesting to note that the apparent failure to construct the Jaffe-Manohar decomposition of $J_{Q C D}^{i}$ arises because of the existence of non-vanishing spatial boundary operator matrix elements, and in the case where $|\Psi\rangle=|0\rangle$, non-vanishing condensates. So physically speaking, it appears that it is the nontrivial vacuum structure of QCD which obstructs the decomposition of distinct quark and gluon observables in this way.

\section{Conclusions}

An important unresolved question in QCD is whether there exist meaningful quark-gluon angular momentum operator decompositions. A common feature of these decompositions is the necessity to ignore spatial boundary operators. However, for quantised field theories it turns out that this is not always the case. By using a more rigorous QFT approach, it is possible though to establish a concrete condition on when these terms vanish. It turns out that a necessary and sufficient condition for this class of operators to vanish is that the operator must annihilate the vacuum state. Applying this condition to the specific case of the boundary operators which feature in the Jaffe-Manohar decomposition, it appears as though at least one of these operators is non-vanishing, and therefore that the decomposition of $J_{Q C D}^{i}$ does not hold. As a result, this means that the Jaffe-Manohar sum rule formed from this decomposition may no longer be valid. From a physical perspective, it seems that the failure of this decomposition, and hence the obstruction to form distinct quark and gluon observables in this way, arises because of the non-trivial vacuum structure of QCD.

\section{Acknowledgements}

This work was supported by the Swiss National Science Foundation (SNF) under contract CRSII2_141847.

\section{References}

[1] M. J. Ashman et al., An investigation of the spin structure of the proton in deep inelastic scattering of polarised muons on polarised protons, Nucl. Phys. B328 (1989) 1.

[2] R. L. Jaffe and A. Manohar, The g 1 problem, Nucl. Phys. B337 (1990) 509.

[3] R. L. Jaffe, Gluon spin in the nucleon, Phys. Lett $\mathbf{B 3 6 5}$ (1996) 359 [hep-ph/9509279].

[4] X. Ji, Gauge-Invariant Decomposition of Nucleon Spin, Phys. Rev. Lett 78 (1997) 610 [hep-ph/9603249].

[5] M. Wakamatsu, Is gauge-invariant complete decomposition of the nucleon spin possible?, Int. J. Mod. Phys. A 29 (2014) 1430012 [hep-ph/1402.4193].

[6] E. Leader and C. Lorcé, The angular momentum controversy: What's it all about and does it matter?, Phys. Rept. 541 (2014) 163 [hep-ph/1309.4235].

[7] P. Lowdon, Boundary terms in quantum field theory and the spin structure of QCD, Nucl. Phys. $\mathbf{B 8 8 9}$ (2014) 801 [hep-th/1408.3233].

[8] R. F. Streater and A. S. Wightman, PCT, Spin and Statistics, and all that, W. A. Benjamin, Inc. (1964). 
[9] R. Haag, Local Quantum Physics, Springer-Verlag (1996).

[10] N. Nakanishi and I. Ojima, Covariant Operator Formalism of Gauge Theories and Quantum Gravity, World Scientific Publishing Co. Pte. Ltd (1990).

[11] F. Strocchi, An Introduction to Non-Perturbative Foundations of Quantum Field Theory, Oxford University Press (2013).

[12] J. Pasupathy and R. K. Singh, Axial Vector Current Matrix Elements and QCD Sum Rules, Int. J. Mod. Phys. A 21 (2006) 5099 [hep-ph/0312304]. 